\title{
Repairing effects of glucosamine sulfate in combination with etoricoxib on articular cartilages of patients with knee osteoarthritis
}

Yong Sun ${ }^{*}$, Changde Wang and Chunzhu Gong

\begin{abstract}
Purpose: To evaluate the repairing effects of glucosamine sulfate combined with etoricoxib on articular cartilages of patients with knee osteoarthritis (KOA).

Methods: A total of $106 \mathrm{KOA}$ patients were randomly divided into control $(n=40)$ and experimental groups $(n=66)$ and treated with etoricoxib alone and glucosamine sulfate plus etoricoxib, respectively. Changes in WOMAC score and clinical efficacy were observed. The synovial fluid was extracted. Bone metabolism indices, growth factors, inflammatory factors, matrix metalloproteinases (MMPs), and NO-induced apoptosis-related factors were measured by ELISA. JNK and Wnt5a mRNA levels were determined using RT-PCR.

Results: After treatment, the total WOMAC scores of both groups significantly declined $(P<0.05)$, being lower in experimental group. The total effective rate of experimental group was higher $(P<0.05)$. BGP and OPG levels rose, especially in experimental group $(P<0.05)$. CTX-II, COMP, and RANKL levels decreased, particularly in experimental group $(P<0.05)$. TGF- $\beta$, IGF-1, and FGF-2 levels increased, especially in experimental group $(P<0.05)$. Both groups, particularly experimental group, had decreased levels of IL-1 $\beta$, IL-17, IL-18, TNF-a, MMP-3, MMP-9, and MMP-13 $(P<0.05)$. JNK and Wnt5a mRNA levels of both groups dropped, which were lower in experimental group $(P<0.05)$. NO and LPO levels reduced, being lower in experimental group. SOD level rose, especially in experimental group $(P<0.05)$.

(Continued on next page)
\end{abstract}

\footnotetext{
* Correspondence: ymggbo@gmail.com

Shenzhen Pingle Orthopedic Hospital (Shenzhen Pingshan Traditional Chinese Medicine Hospital), 40 Jintang Street, Luohu District, Shenzhen

518010, Guangdong Province, People's Republic of China
}

(c) The Author(s). 2020 Open Access This article is licensed under a Creative Commons Attribution 4.0 International License, which permits use, sharing, adaptation, distribution and reproduction in any medium or format, as long as you give appropriate credit to the original author(s) and the source, provide a link to the Creative Commons licence, and indicate if changes were made. The images or other third party material in this article are included in the article's Creative Commons licence, unless indicated otherwise in a credit line to the material. If material is not included in the article's Creative Commons licence and your intended use is not permitted by statutory regulation or exceeds the permitted use, you will need to obtain permission directly from the copyright holder. To view a copy of this licence, visit http://creativecommons.org/licenses/by/4.0/ The Creative Commons Public Domain Dedication waiver (http://creativecommons.org/publicdomain/zero/1.0/) applies to the data made available in this article, unless otherwise stated in a credit line to the data. 
(Continued from previous page)

Conclusion: Glucosamine sulfate plus etoricoxib can repair the articular cartilages of KOA patients. Probably, JNK and Wnt5a are downregulated to inhibit the secretion of MMPs through lowering the levels of inflammatory factors, thereby delaying cartilage matrix degradation. NO-induced chondrocyte apoptosis may be suppressed via the SOD pathway.

Keywords: Cartilage, Etoricoxib, Glucosamine sulfate, Knee osteoarthritis, Repair

\section{Background}

Knee osteoarthritis (KOA) is a chronic disease of retrograde degeneration of articular cartilage and secondary hyperostosis, which frequently occurs in middle-aged and elderly people, being more common in women. In China, OA occurs in about $3 \%$ of people, dominated by KOA. X-ray features of KOA are found in about $60 \%$ of people aged above 55 years, and its incidence rate is up to $85 \%$ in people aged above 65 [1]. With the prolongation of life expectancy and the improvement of quality of life, KOA has aroused increasing attention.

$\mathrm{KOA}$ is clinically manifested as joint stiffness, pain, local swelling, deformity, and dysfunction in different degrees. The pathological features of KOA are progressive destruction and degeneration of articular cartilage, subchondral osteosclerosis or cystic changes, joint synovial hyperplasia, osteophyte formation at the joint edge, contracture or hypertrophy of the joint capsule, and contracture or relaxation of ligament [2]. The pathological mechanism of KOA remains unclear, and there still lacks specific therapeutic methods to reduce pain and control disease progression currently. Therefore, selecting drug or non-drug therapy based on the patients' conditions is the principle of treatment currently.

Glucosamine sulfate, as a natural amino acid monosaccharide, can supplement the cartilage matrix, delay the cartilage degradation, and promote the synthesis of proteoglycan in chondrocytes, which is a nutritional drug for cartilage [3]. Glucosamine sulfate is able to alleviate the symptoms of joint pain, delay and alter the pathological process of KOA, specifically supply the cartilage matrix in articular cartilage, and restore the normal metabolism. Thus, it is the only effective drug that can block the vicious pathological circle of KOA and promote the cartilage repair currently. As a specific inhibitor of cyclooxygenase-2 (COX-2) and a non-steroidal anti-inflammatory drug, etoricoxib has analgesic, antiinflammatory, and antipyretic effects [4], which has been widely used to relieve pain, reduce morning stiffness, and improve the joint function of $\mathrm{OA}$ and rheumatoid arthritis patients. In this study, glucosamine sulfate in combination with etoricoxib were used to treat KOA, and the mechanism for articular cartilage repair was explored, aiming to provide references for clinical KOA treatment.

\section{Materials and methods}

\section{Subjects}

This study was approved by the ethics committee of our hospital (approval no. SPOH201601003), and written informed consent was obtained from all patients. A total of $106 \mathrm{KOA}$ patients treated in our hospital from January 2016 to May 2019 were selected, including 23 males and 83 females aged $48-75$ years with the disease courses of $1-5$ years. The patients were numbered in accordance with the sequence of hospitalization and randomly divided into a control group $(n=40)$ and an experimental group $(n=66)$. In the control group, there were 9 males and 31 females with a mean age of $62.07 \pm 11.32$ years. The mean course of disease was $3.59 \pm 0.75$ months. In terms of the lesion site, there were 18 cases in the left knee and 22 cases in the right knee. In terms of the Kellgren-Lawrence classification, there were 9 cases of grade I, 15 cases of grade II, and 16 cases of grade III. In the experimental group, there were 14 males and 52 females with a mean age of $61.58 \pm 10.24$ years. The mean course of disease was $3.74 \pm 0.89$ months. In terms of the lesion site, there were 35 cases in the left knee and 31 cases in the right knee. In terms of the Kellgren-Lawrence classification, there were 16 cases of grade I, 27 cases of grade II, and 23 cases of grade III. The two groups had comparable baseline clinical data (Table 1).

\section{Related criteria}

The diagnostic criteria of the American College of Rheumatology were employed [5]: (1) aged above 40 years, (2) pain in the knee most of the time within 1 month, (3) morning stiffness for shorter than $30 \mathrm{~min}$, (4) friction sound of joint during movement, (5) OA symptoms found in synovial fluid test, and (6) osteophyte formation at the joint edge shown in X-ray image. Those who meet the criteria (1), (2), (3), and (4); or (2) and (6); or (2), (3), (4), and (5) were diagnosed as KOA. KOA was graded based on the X-ray image of knee joint according to the KellgrenLawrence diagnostic criteria [6]: Grade 0 (normal), grade I (suspicious narrowing of joint space, possibly with osteophytes), grade II (suspicious narrowing of joint space, with obvious osteophytes), grade III (obvious narrowing and sclerosis of joint space, with moderate osteophyte formation), and grade IV (obvious narrowing, sclerosis and 
Table 1 Baseline clinical data of subjects $(\bar{x} \pm s)[n(\%)]$

\begin{tabular}{|c|c|c|c|c|}
\hline Item & Experimental group $(n=40)$ & Control group $(n=40)$ & $t / x^{2}$ & $P$ \\
\hline Gender (case) & & & 0.024 & 0.876 \\
\hline Male & 14 & 9 & & \\
\hline Female & 52 & 31 & & \\
\hline Age (year) & $61.58 \pm 10.24$ & $62.07 \pm 11.32$ & 0.229 & 0.819 \\
\hline Disease course (year) & $3.74 \pm 0.89$ & $3.59 \pm 0.75$ & 0.891 & 0.375 \\
\hline Lesion site & & & 0.642 & 0.423 \\
\hline Left & 35 & 18 & & \\
\hline Right & 31 & 22 & & \\
\hline Kellgren-Lawrence grade & & & 0.285 & 0.867 \\
\hline । & 16 & 9 & & \\
\hline$\|$ & 27 & 15 & & \\
\hline III & 23 & 16 & & \\
\hline
\end{tabular}

deformity of joint space, with massive osteophyte formation).

Inclusion criteria include (1) patients meeting the above diagnostic criteria, with lesions on single knee; (2) those without an allergic history to glucosamine sulfate or etoricoxib; and (3) those who cooperated willingly in this study. Exclusion criteria include (1) Patients complicated with diseases of the endocrine system, digestive system, blood system, heart, liver, kidney, or tumors; (2) those complicated with congenital limb deformity, skin disease, or acute trauma of local knee; (3) those with rheumatoid arthritis, septic arthritis, traumatic arthritis, gout, ankylosing spondylitis, or other rheumatic diseases; (4) those who used corticoids and non-steroidal antiinflammatory drugs, or immune enhancers, inhibitors, modulators or other decoctions and Chinese patent medicine, or underwent physical therapy; (5) those with mental disorders or disturbance of consciousness; and (6) those who had missing or incomplete medical data, or quit halfway.

\section{Treatment methods}

For the control group, etoricoxib (Merck Sharp \& Dohme (Australia) Pty. Ltd., import drug license H20120130, $60 \mathrm{mg} \times 5$ tablets) was orally taken once a day $(60 \mathrm{mg} /$ time $)$ for 6 weeks. For the experimental group, glucosamine sulfate capsules (Rottapharm Ltd., import drug license H20170108, $0.25 \mathrm{~g} \times 20$ capsules) were orally taken 3 times a day $(0.5 \mathrm{~g} /$ time $)$ for 6 weeks based on the treatment of the control group.

\section{Evaluation of therapeutic effects}

The knee function was evaluated before and after treatment using the Western Ontario and McMaster Universities Arthritis Index (WOMAC). The scale consists of 24 items in 3 parts. Each score has 4 grades: 0 point (no difficulty), 1 point (mild), 2 points (moderate), 3 points (severe), and 4 points (extremely severe), and the total score is 96 points. There are 5 items for pain (total score of 20 points), 2 items for joint stiffness (total score of 8 points), and 17 items for joint function (total score of 68 points). A higher score corresponds to severer KOA in patients.

The clinical efficacy was determined as follows. Ineffective: Such clinical symptoms as swelling and pain have no changes after treatment, and the knee function has no improvement. Effective: Knee pain occurs sometimes, there is mild pain in walking, the knee function is basically restored, and it is a little uncomfortable to walk up and down stairs. Markedly effective: There is no knee pain at rest, but sometimes pain in activity, the joint function is obviously restored, and neither work nor life is affected. Clinically controllable: The clinical symptoms (swelling and pain in the knee joint) and signs completely disappear, and the joint function returns to normal. Total effective rate $=$ (clinically controllable + markedly effective + effective) $/$ total cases $\times 100 \%$.

\section{Detection indices}

After iodophor disinfection, the articular cavity was punctured via the lateral patellar approach in a supine position, and the synovial fluid was extracted and stored in a sterile tube. Bone metabolism indices (bone gamma-carboxy glutamic acid-containing protein (BGP), orthopantomography (OPG), crosslinked c-telopeptide of type II collagen (CTXII), cartilage oligomeric matrix protein (COMP), and cell nuclear factor $\mathrm{\kappa B}$ acceptor activating factor ligand (RANKL)), growth factors (transforming growth factor- $\beta$ (TGF- $\beta$ ), insulin-like growth factor-1 (IGF-1), and fibroblast growth factor-2 (FGF-2)), inflammatory factors (interleukin-1 $\beta$ (IL$1 \beta)$, IL-17, IL-18, and tumor necrosis factor- $\alpha$ (TNF- $\alpha)$ ), matrix metalloproteinases (matrix metalloproteinase-3 (MMP-3), MMP-9, and MMP-13), and NO-induced apoptosis-related factors (nitric oxide (NO), superoxide dismutase (SOD), and lipid peroxidase (LPO)) were measured by ELISA. Total RNA of the synovial fluid was extracted by 
the Trizol method and reverse-transcribed. RT-qPCR was carried out to detect the mRNA expression levels of C-Jun N-terminal kinase (JNK) and Wnt family member 5a (Wnt5a). Primer sequences for JNK are FP, 5'-CGGGAT CTTCAACTTTAACAT GGAAGTGCTTTCTGTGACTT TAAA-3' and RP, 5'-CCCAAGCTTACTCCTACTAAAA AGCACTTACTTTTAAAGTC-3'. Primer sequences for Wnt5a are FP, 5'-CACACACTACATCAGTGGCTCA AAG-3' and RP, 5' -TCCAGCACATGAACGTGTAAACAG-3'. Meanwhile, GAPDH (FP, 5' CTTTAACATGGA AGTGCGGGA-3'; RP, 5' -CTAAAAAGCACTTACCCC AAGCTATC-3') was utilized as the internal reference. Each sample was tested three times independently.

\section{Statistical analysis}

All data were statistically analyzed by the SPSS 19.0 software. The numerical data were expressed as $[n(\%)]$ and subjected to the $x^{2}$ test. The measurement data were represented as mean \pm standard deviation $(\bar{x} \pm s)$. The comparisons between two groups were performed by the independent $t$ test, and those at different points were conducted with the paired $t$ test. $P<0.05$ was considered statistically significant.

\section{Results}

WOMAC scores

The pain, joint stiffness, joint function scores, and total WOMAC score of the two groups significantly declined after treatment compared with those before treatment $(P$ $<0.05)$. After treatment, each score and total WOMAC score of the experimental group were lower than those of the control group $(P<0.05)$ (Table 2$)$.

\section{Clinical effective rates}

The total effective rate of the experimental group was higher than that of the control group $(92.42 \%$ vs. $67.50 \%, P<0.05$ ) (Table 3 ).

\section{Bone metabolism indices}

The levels of BGP and OPG significantly rose after treatment compared with those before treatment in both groups, which were higher in the experimental group than in the control group $(P<0.05)$. The levels of CTXII, COMP, and RANKL significantly decreased after treatment compared with those before treatment in both groups, which were lower in the experimental group than in the control group $(P<0.05)$ (Table 4$)$.

\section{Growth factors}

The levels of TGF- $\beta$, IGF-1, and FGF-2 were significantly higher in both groups after treatment than those before treatment, being higher in the experimental group $(P<0.05)$ (Table 5).

\section{Inflammatory factors and MMPs}

Both groups had significantly decreased levels of IL-1 $\beta$, IL-17, IL-18, TNF- $\alpha$, MMP-3, MMP-9, and MMP-13 after treatment compared with those before treatment, being lower in the experimental group $(P<0.05)$ (Table 6).

\section{JNK and Wnt5a mRNA levels}

There were lower mRNA levels of JNK and Wnt5a in both groups after treatment than those before treatment, which were significantly lower in the experimental group than in the control group $(P<0.05)$ (Fig. 1$)$.

\section{NO-induced apoptosis-related factors}

Compared with before treatment, the levels of NO and LPO reduced after treatment, which were lower in the

Table 2 WOMAC scores $(\bar{x} \pm s)$

\begin{tabular}{llll} 
Item & Experimental group $(n=66)$ & Control group $(n=40)$ & $t$ \\
\hline Pain & & & $P$ \\
Before treatment & $17.36 \pm 2.59$ & $17.18 \pm 2.45$ & 0.354 \\
After treatment & $8.94 \pm 0.83^{\mathrm{ab}}$ & $12.57 \pm 1.36^{\mathrm{a}}$ & 17.086 \\
Joint stiffness & & & 0.724 \\
Before treatment & $7.21 \pm 0.68$ & $7.25 \pm 0.72$ & 0.287 \\
After treatment & $3.14 \pm 0.33^{\mathrm{ab}}$ & $5.07 \pm 0.49^{\mathrm{a}}$ & 24.224 \\
Joint function & & & 0.775 \\
Before treatment & $57.83 \pm 6.07$ & $57.36 \pm 5.91$ & 0.000 \\
After treatment & $30.62 \pm 2.89^{\mathrm{ab}}$ & $41.27 \pm 4.08^{\mathrm{a}}$ & 0.390 \\
Total score & & & 0.697 \\
Before treatment & $84.16 \pm 10.25$ & $84.32 \pm 10.43$ & 0.000 \\
After treatment & $41.83 \pm 4.09^{\mathrm{ab}}$ & $63.57 \pm 7.38^{\mathrm{a}}$ & 0.077 \\
\hline
\end{tabular}


Table 3 Clinical effective rates

\begin{tabular}{llll}
\hline Item & Experimental group $(n=66)$ & Control group $(n=40)$ & $x^{2}$ \\
\hline Clinically controllable (case) & 43 & 9 & $P$ \\
Markedly effective (case) & 9 & 8 & 10 \\
Effective (case) & 9 & 13 & 20.770 \\
Ineffective (case) & 5 & 67.50 & 0.000 \\
Total effective rate (\%) & 92.42 &
\end{tabular}

experimental group. The level of SOD rose after treatment, being higher in the experimental group $(P<0.05)$ (Table 7).

\section{Discussion}

KOA is a chronic injury of knee joint cartilage caused by factors such as long-term load bearing and mechanical damage [7]. With the increase of age, the degradation of fibrous tissue, osteoporosis, and changes of synovial fluid composition become more serious resulting in the deficiency of joint cartilage nutrition, the reduction of cartilage lubrication, and the increase of pressure on the bone and articular surface for chronic strain and progressive damage [8]. Meanwhile, osteoproliferation causes osteophytes at the edge of the joint, leading to increased bone pressure, which in turn affects blood circulation, aggravates the degree of injury of articular cartilage, and finally causes symptoms such as swelling, pain, and even dysfunction. Deformation and loss of articular cartilage, regeneration of subchondral bone, and marginal bone are important features of $\mathrm{KOA}$, and articular cartilage is the initial site of the disease. Therefore, in the course of treatment, it is necessary to promote the repair of cartilage tissue while ensuring pain relief and disease progression under control [9]. Glucosamine in human articular cartilage is a basic substance needed in the synthesis of aminoglycans. Oral glucosamine sulfate can directly supplement the cartilage matrix, slow down cartilage degradation, facilitate the synthesis of cartilage protein and the recovery of chondrocyte matrix secretion, and in turn improve the articular cartilage structure [10]. Etoricoxib can selectively inhibit the synthesis of cyclooxygenase and prostaglandin and play an anti-inflammatory role, so as to effectively alleviate joint swelling and pain [11]. The results of this study showed that compared with before treatment, pain, joint stiffness, joint function scores, and total scores of the WOMAC scale were significantly reduced in the two groups after treatment $(P<$ 0.05), indicating that both glucosamine sulfate combined with etoricoxib and etoricoxib alone had significant effects. After treatment, the scores of the experimental group and the total score of WOMAC were lower than those of the control group $(P<0.05)$, suggesting that the efficacy of glucosamine sulfate combined with etoricoxib

Table 4 Bone metabolism indices $(\bar{x} \pm s)$

\begin{tabular}{|c|c|c|c|c|}
\hline Item & Experimental group $(n=66)$ & Control group $(n=40)$ & $t$ & $P$ \\
\hline \multicolumn{5}{|l|}{$\mathrm{BGP}(\mathrm{ng} / \mathrm{mL})$} \\
\hline Before treatment & $3.39 \pm 0.58$ & $3.42 \pm 0.61$ & 0.253 & 0.801 \\
\hline After treatment & $5.78 \pm 1.26^{\mathrm{ab}}$ & $4.13 \pm 0.94^{\mathrm{a}}$ & 7.157 & 0.000 \\
\hline \multicolumn{5}{|l|}{$\mathrm{OPG}(\mathrm{pg} / \mathrm{mL})$} \\
\hline Before treatment & $118.42 \pm 12.37$ & $120.51 \pm 12.64$ & 0.836 & 0.405 \\
\hline After treatment & $232.85 \pm 24.19^{\mathrm{ab}}$ & $163.79 \pm 17.38^{\mathrm{a}}$ & 15.747 & 0.000 \\
\hline \multicolumn{5}{|l|}{ CTX-II } \\
\hline Before treatment & $2.36 \pm 0.27$ & $2.41 \pm 0.29$ & 0.899 & 0.371 \\
\hline After treatment & $0.94 \pm 0.08^{\mathrm{ab}}$ & $1.62 \pm 0.18^{\mathrm{a}}$ & 18.569 & 0.000 \\
\hline \multicolumn{5}{|l|}{ COMP } \\
\hline Before treatment & $4.85 \pm 0.62$ & $4.90 \pm 0.67$ & 0.390 & 0.697 \\
\hline After treatment & $2.59 \pm 0.31^{a b}$ & $3.46 \pm 0.48^{a}$ & 11.345 & 0.000 \\
\hline \multicolumn{5}{|l|}{ RANKL } \\
\hline Before treatment & $84.73 \pm 9.69$ & $83.82 \pm 9.44$ & 0.473 & 0.637 \\
\hline After treatment & $54.38 \pm 5.77^{\mathrm{ab}}$ & $72.95 \pm 7.51^{\mathrm{a}}$ & 14.307 & 0.000 \\
\hline
\end{tabular}

Compared with before treatment, ${ }^{a} P<0.05$; compared with control group, ${ }^{\mathrm{b}} P<0.05$. BGP bone gamma-carboxy glutamic acid-containing protein, COMP cartilage oligomeric matrix protein, CTX-II crosslinked c-telopeptide of type II collagen, OPG orthopantomography, RANKL cell nuclear factor $\mathrm{KB}$ acceptor activating factor ligand 
Table 5 Growth factors $(\bar{x} \pm s)$

\begin{tabular}{|c|c|c|c|c|}
\hline Item & Experimental group $(n=66)$ & Control group $(n=40)$ & $t$ & $P$ \\
\hline \multicolumn{5}{|l|}{ TGF- $\beta$ ( $\mu \mathrm{g} / \mathrm{L})$} \\
\hline Before treatment & $22.07 \pm 2.16$ & $21.93 \pm 2.08$ & 0.328 & 0.744 \\
\hline After treatment & $30.15 \pm 3.04^{\mathrm{ab}}$ & $25.42 \pm 2.47^{\mathrm{a}}$ & 8.313 & 0.000 \\
\hline \multicolumn{5}{|l|}{ IGF-1 ( $\mu \mathrm{g} / \mathrm{L})$} \\
\hline Before treatment & $83.41 \pm 10.29$ & $82.87 \pm 9.83$ & 0.266 & 0.791 \\
\hline After treatment & $95.72 \pm 12.06^{\mathrm{ab}}$ & $89.64 \pm 11.25^{\mathrm{a}}$ & 2.579 & 0.011 \\
\hline \multicolumn{5}{|l|}{$\mathrm{FGF}-2$ (ng/L) } \\
\hline Before treatment & $23.58 \pm 2.47$ & $24.01 \pm 2.53$ & 0.861 & 0.391 \\
\hline After treatment & $34.64 \pm 3.59^{\mathrm{ab}}$ & $28.39 \pm 2.92^{\mathrm{a}}$ & 9.298 & 0.000 \\
\hline
\end{tabular}

Compared with before treatment, ${ }^{\mathrm{a}} P<0.05$; compared with control group, ${ }^{\mathrm{b}} P<0.05$. FGF-2 fibroblast growth factor-2, IGF-1 insulin-like growth factor-1, TGF- $\beta$ transforming growth factor- $\beta$

was better than that of etoricoxib alone. The total effective rate of the experimental group was significantly higher than that of the control group $(P<0.05)$, which was consistent with the above results.

BGP, which is secreted by osteoblasts and mainly deposited in bone matrix, is a kind of non-specific collagen that promotes bone mineralization. It is released in large quantities when bone matrix is degraded, which can reflect the activity of osteoblasts [12]. OPG/RANKL/ RANK plays a regulatory role in the process of bone formation and absorption [13]. OPG can inhibit the differentiation and maturation of osteoclasts. The maturation and proliferation of RANK and its ligand osteoclasts promote the maturation and proliferation of osteoclasts. The increase in the RANKL/OPG ratio indicates that the imbalance and abnormality of bone reconstruction

Table 6 Inflammatory factors and MMPs $(\bar{x} \pm s)$

\begin{tabular}{|c|c|c|c|c|}
\hline Item & Experimental group $(n=66)$ & Control group $(n=40)$ & $t$ & $P$ \\
\hline \multicolumn{5}{|l|}{$\mathrm{IL}-1 \beta(\mathrm{pg} / \mathrm{mL})$} \\
\hline Before treatment & $74.37 \pm 6.95$ & $75.02 \pm 7.14$ & 0.462 & 0.645 \\
\hline After treatment & $38.56 \pm 3.74^{\mathrm{ab}}$ & $51.48 \pm 4.89^{\mathrm{a}}$ & 15.322 & 0.000 \\
\hline \multicolumn{5}{|l|}{ IL-17 ( $\mu \mathrm{g} / \mathrm{L})$} \\
\hline Before treatment & $391.64 \pm 40.25$ & $389.73 \pm 39.52$ & 0.238 & 0.812 \\
\hline After treatment & $205.38 \pm 19.76^{\mathrm{ab}}$ & $276.41 \pm 26.11^{\mathrm{a}}$ & 15.858 & 0.000 \\
\hline \multicolumn{5}{|l|}{ IL-18 (pg/mL) } \\
\hline Before treatment & $232.59 \pm 24.16$ & $241.25 \pm 23.96$ & 1.794 & 0.076 \\
\hline After treatment & $148.73 \pm 13.25^{\mathrm{ab}}$ & $184.67 \pm 17.13^{\mathrm{a}}$ & 12.099 & 0.000 \\
\hline \multicolumn{5}{|l|}{ TNF-a $(\mathrm{pg} / \mathrm{mL})$} \\
\hline Before treatment & $87.94 \pm 9.27$ & $88.31 \pm 9.56$ & 0.197 & 0.844 \\
\hline After treatment & $30.52 \pm 2.86^{\mathrm{ab}}$ & $52.45 \pm 5.02^{\mathrm{a}}$ & 28.679 & 0.000 \\
\hline \multicolumn{5}{|l|}{ MMP-3 (ng/ml) } \\
\hline Before treatment & $217.93 \pm 20.54$ & $221.01 \pm 21.18$ & 0.739 & 0.461 \\
\hline After treatment & $98.46 \pm 9.75^{a b}$ & $158.37 \pm 14.82^{\mathrm{a}}$ & 21.359 & 0.000 \\
\hline \multicolumn{5}{|l|}{ MMP-9 (ng/ml) } \\
\hline Before treatment & $66.83 \pm 7.14$ & $67.25 \pm 7.21$ & 0.292 & 0.770 \\
\hline After treatment & $30.26 \pm 2.97^{\mathrm{ab}}$ & $45.38 \pm 4.62^{\mathrm{a}}$ & 20.524 & 0.000 \\
\hline \multicolumn{5}{|l|}{ MMP-13 (ng/ml) } \\
\hline Before treatment & $275.18 \pm 28.09$ & $274.92 \pm 27.83$ & 0.046 & 0.963 \\
\hline After treatment & $152.43 \pm 14.72^{\mathrm{ab}}$ & $193.76 \pm 18.69^{a}$ & 10.987 & 0.000 \\
\hline
\end{tabular}

Compared with before treatment, ${ }^{a} P<0.05$; compared with control group, ${ }^{b} P<0.05$. IL interleukin, MMP matrix metalloproteinase, TNF- $a$ tumor necrosis factor-a 

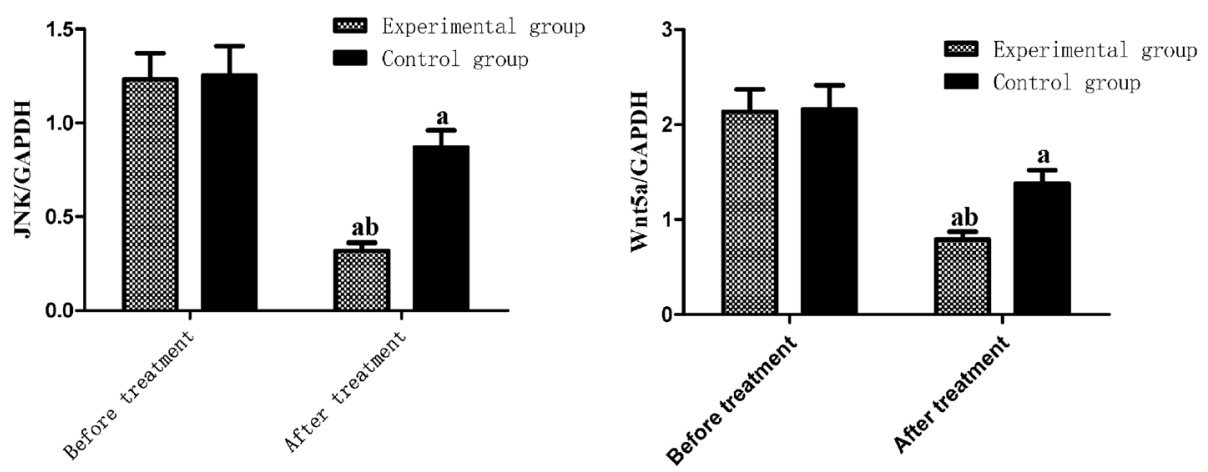

Fig. $1 \mathrm{JNK}$ and Wnt5a mRNA levels. Compared with before treatment, ${ }^{a} P<0.05$; compared with control group, ${ }^{\mathrm{b}} P<0.05$. JNK, C-Jun N-terminal kinase; Wnt5a, Wnt family member $5 a$

can also lead to bone loss and bone density reduction [14]. CTX-II, a small polypeptide mainly distributed in cartilage, can be used to reflect the degradation of collagen type II by protease in cartilage. With rising level of CTX-II, the joint damage was aggravated [15]. As a sensitive marker of articular cartilage injury, COMP, which is mainly expressed in cartilage, has high tissue specificity, and its level is positively correlated with the degree of joint injury [16]. Herein, compared with before treatment, BGP and OPG were increased significantly after treatment, and those of the experimental group were significantly higher than those of the control group $(P<$ 0.05). CTX-II, COMP, and RANKL were significantly reduced, and those of the experimental group were significantly lower than those of the control group $(P<0.05)$. Thus, glucosamine sulfate combined with etoricoxib in the treatment of KOA can better regulate the bone metabolism indexes of patients, delay or hinder joint degeneration, and improve joint function.

TGF- $\beta$ is a crucial cartilage repair factor, which can maintain the normal structure and function of articular cartilage. The higher the level of TGF- $\beta$ is, the more favorable the repair of knee osteoarticular injury will be
[17]. IGF-1 can accelerate the proliferation of chondrocytes and the formation of chondrocyte colonies by stimulating chondrocytes to synthesize protein polysaccharides and collagen type II. The expression level of IGF-1 is positively correlated with the response of cartilage repair [18]. FGF-2, an effective angiogenic factor in vivo, can promote the generation of new blood vessels by inducing vascular endothelial cells. In addition, as a mitogen and morphogenetic factor of chondrocytes, FGF-2 can promote the repair of cartilage and bone tissue [19]. This study found that compared with before treatment, the levels of TGF- $\beta$, IGF- 1 , and FGF- 2 in the two groups were significantly higher than those in the control group after treatment, and those of the experimental group were significantly higher than those of the control group $(P<0.05)$. Accordingly, glucosamine sulfate and etoricoxib can repair the cartilage tissue of KOA patients, and the combination of the two drugs has a better repair effect.

Inflammatory response is the main pathological feature of KOA; inflammatory factors are important factors that damage joint cartilage and cause swelling and pain, and inflammatory factors mainly play a role through a

Table 7 NO-induced apoptosis-related factors $(\bar{x} \pm s)$

\begin{tabular}{|c|c|c|c|c|}
\hline Item & Experimental group $(n=66)$ & Control group $(n=40)$ & $t$ & $P$ \\
\hline \multicolumn{5}{|l|}{$\mathrm{NO}(\mu \mathrm{mol} / \mathrm{L})$} \\
\hline Before treatment & $65.82 \pm 7.14$ & $66.03 \pm 7.21$ & 0.146 & 0.884 \\
\hline After treatment & $27.54 \pm 2.69^{\mathrm{ab}}$ & $42.39 \pm 4.28^{a}$ & 21.957 & 0.000 \\
\hline \multicolumn{5}{|l|}{$\mathrm{SOD}(\mathrm{U} / \mathrm{mL})$} \\
\hline Before treatment & $69.41 \pm 6.87$ & $70.12 \pm 6.93$ & 0.514 & 0.608 \\
\hline After treatment & $118.59 \pm 12.16^{\mathrm{ab}}$ & $89.48 \pm 9.04^{a}$ & 13.096 & 0.000 \\
\hline \multicolumn{5}{|l|}{ LPO (nmol/mL) } \\
\hline Before treatment & $8.74 \pm 0.83$ & $8.81 \pm 0.86$ & 0.415 & 0.679 \\
\hline After treatment & $1.06 \pm 0.15^{\mathrm{ab}}$ & $3.69 \pm 0.42^{\mathrm{a}}$ & 46.343 & 0.000 \\
\hline
\end{tabular}


series of cascade amplification reactions [20]. IL-1 $\beta$ can promote the secretion of MMPs, which leads to the degradation of cartilage matrix and chondrocyte apoptosis. IL-17 exerts a strong induction effect on the catabolism of chondrocytes, thus promoting cartilage degradation, and in addition, it can stimulate the production of IL-6 and indirectly promote the secretion of MMPs [21]. IL18 can induce the production of nitric oxide and prostaglandin E2, and then participate in the process of KOA inflammation and joint injury [22]. TNF- $\alpha$ can activate and aggregate leukocytes, inhibit the synthesis of type II collagen and proteoglycan, inhibit the self-repair of cartilage, and promote cartilage degradation. MMPs can degrade extracellular matrix of articular cartilage [23, 24]. MMP-3 secreted by synovial cells and chondrocytes can not only degrade matrix protein substrates in a variety of extracellular matrix but also activate enzymes such as MMP-9 and MMP-13 to produce a cascade amplification reaction. MMP-9 can degrade collagen by destroying the reticular structure formed by cartilage matrix and collagen. The synergistic effect of MMP-3 and MMP-9 can accelerate the destruction process and make the changes of collagen and cartilage irreversible. At present, MMP-13, the most effective type II collagen degrading enzyme, can degrade all kinds of collagen and directly damage the integrity of articular cartilage. This study showed that after treatment, the levels of IL-1 $\beta$, IL-17, IL-18, TNF- $\alpha$, MMP-3, MMP-9, and MMP-13 in the two groups were significantly lower than those before treatment $(P<0.05)$. After treatment, the levels in the experimental group were lower than those in the control group $(P<0.05)$. Hence, glucosamine sulfate and etoricoxib can inhibit the secretion of inflammatory factors and then reduce the levels of MMPs, so as to slow down the degradation of cartilage matrix and facilitating the repair of damaged chondrocytes. In the process of arthritis cartilage destruction, IL-1 $\beta$ can upregulate the expression of Wnt5a which can upregulate the expression of matrix metalloproteinase through the mediation of JNK signaling pathway [25]. The mRNA levels of JNK and Wnt5a were detected in this study, which were lower after treatment than those before treatment, and those of the experimental group were lower than those of the control group $(P<0.05)$. The results indicated that JNK and Wnt5a were involved in the process of decreasing IL- $1 \beta$ and inhibiting matrix metalloproteinase in the treatment of KOA with glucosamine sulfate and etoricoxib.

As an important pathway leading to chondrocyte apoptosis, NO-induced apoptosis is closely related to NO, LPO, and SOD [26]. A large number of free radicals produced by the body during the onset of KOA can promote apoptosis [27]. NO, a key member of free radicals, can aggravate chondrocyte injury by inducing chondrocyte apoptosis. LPO, the metabolite of free radical lipid peroxidation, can damage the structure of cells and cell membranes. This indicator is often used to indirectly reflect the damage degree of free radicals to tissue cells. SOD can remove oxygen free radicals through disproportionation and block the cytochrome C-dependent mitochondrial apoptosis pathway, thereby playing a protective role in the synovium and chondrocytes. In this study, compared with before treatment, NO and LPO levels were decreased after treatment, and those of the experimental group were lower than those of the control group. Moreover, the SOD level was increased and that of the experimental group was higher than that of the control group $(P<0.05)$. We postulated that glucosamine sulfate combined with etoricoxib dominated in the repair of KOA cartilage by increasing SOD level, inhibiting lipid peroxidation, reducing free radicals, inhibiting apoptosis of chondrocytes, and ultimately protecting and repairing cartilage.

\section{Conclusions}

In summary, glucosamine sulfate combined with etoricoxib may reduce the expression of JNK and Wnt5a to inhibit the secretion of matrix metalloproteinase, and then slow down the degradation of cartilage matrix. It may also inhibit the NO-induced chondrocyte apoptosis through SOD pathway. In the future, we will build animal models or study at the cellular level to further verify this conclusion.

\section{Abbreviations}

BGP: Bone gamma-carboxy glutamic acid-containing protein:

COMP: Cartilage oligomeric matrix protein; COX-2: Cyclooxygenase-2; CTXII: Crosslinked c-telopeptide of type II collagen; FGF-2: Fibroblast growth factor-2; IGF-1: Insulin-like growth factor-1; IL: Interleukin; JNL: C-Jun Nterminal kinase; KOA: Knee osteoarthritis; LPO: Lipid peroxidase; MMP: Matrix metalloproteinase; NO: Nitric oxide; OA: Osteoarthritis;

OPG: Orthopantomography; RANKL: Cell nuclear factor KB acceptor activating factor ligand; SOD: Superoxide dismutase; TGF- $\beta$ : Transforming growth factor- $\beta$; TNF-a: Tumor necrosis factor- $a$; Wnt5a: Wnt family member $5 a$; WOMAC: Western Ontario and McMaster Universities Arthritis Index

\section{Acknowledgements \\ We thank all coauthors for their significant contributions.}

\section{Authors' contributions}

YS: study design, acquisition of participants and data, analysis and interpretation of data, and preparation of manuscript. CW: acquisition of participants and data and analysis and interpretation of data. CG: acquisition of participants and data and analysis and interpretation of data. All authors approve the manuscript submission.

\section{Funding}

This study was not financially supported.

Availability of data and materials

The datasets used and/or analyzed during the current study are available either online or from the corresponding author on reasonable request. 


\section{Ethics approval and consent to participate}

This study was approved by the ethics committee of Shenzhen Pingle Orthopaedic Hospital and performed in accordance with the ethical standards as laid down in the 1964 Declaration of Helsinki. Informed written consent was obtained from all individual participants.

\section{Consent for publication}

Not applicable.

\section{Competing interests}

The authors declare that they have no competing interests

Received: 5 March 2020 Accepted: 25 March 2020

Published online: 16 April 2020

\section{References}

1. Kang SH, Lee SJ, Press JM, Zhang LQ. Real-time three-dimensional knee moment estimation in knee osteoarthritis: toward biodynamic knee osteoarthritis evaluation and training. IEEE Trans Neural Syst Rehabil Eng. 2019;27:1263-72.

2. Dell'Isola A, Steultjens M. Classification of patients with knee osteoarthritis in clinical phenotypes: data from the osteoarthritis initiative. PLoS One. 2018; 13:e0191045.

3. Bruyère $O$, Altman RD, Reginster JY. Efficacy and safety of glucosamine sulfate in the management of osteoarthritis: evidence from real-life setting trials and surveys. Semin Arthritis Rheum. 2016;45:S12-7.

4. Z Zhang S, Zhang Y, Liu P, Zhang W, Ma JL, Wang J. Efficacy and safety of etoricoxib compared with NSAIDs in acute gout: a systematic review and a meta-analysis. Clin Rheumatol. 2016;35:151-8.

5. Au KY, Chen H, Lam WC, Chong CO, Lau A, Vardhanabhuti V, et al. Sinew acupuncture for knee osteoarthritis: study protocol for a randomized shamcontrolled trial. BMC Complement Altern Med. 2018;18:133.

6. Charlton JM, Krowchuk NM, Hatfield GL, Guenette JA, Hunt MA. Contralateral limb foot rotation during unilateral toe-in or toe-out walking in people with knee osteoarthritis. Gait Posture. 2018;62:132-4.

7. Steultjens M. KNEEMO-Prevention and personalized treatments in knee osteoarthritis: an Initial Training Network-FP7. Impact. 2017:2017:69-71.

8. Maudens $P$, Jordan O, Allémann E. Recent advances in intra-articular drug delivery systems for osteoarthritis therapy. Drug Discov Today. 2018;23: 1761-75.

9. Zhang W, Ouyang $H$, Dass $C R, X u$ J. Current research on pharmacologic and regenerative therapies for osteoarthritis. Bone Res. 2016;4:15040.

10. Eaton CB, Sayeed M, Ameernaz S, Roberts MB, Maynard JD, Driban JB, et al. Sex differences in the association of skin advanced glycation endproducts with knee osteoarthritis progression. Arthritis Res Ther. 2017;19:36.

11. Feng $X$, Beiping $L$. Therapeutic efficacy of ozone injection into the knee for the osteoarthritis patient along with oral celecoxib and glucosamine. J Clin Diagn Res. 2017;11:UC01-3.

12. Xing D, Kwong J, Yang Z, Hou Y, Zhang W, Ma B, et al. Intra-articular injection of mesenchymal stem cells in treating knee osteoarthritis: a systematic review of animal studies. Osteoarthritis Cartilage. 2018;26:445-61.

13. Amin N, Boccardi V, Taghizadeh M, Jafarnejad S. Probiotics and bone disorders: the role of RANKL/RANK/OPG pathway. Aging Clin Exp Res. 2020; 32:363-71.

14. Shruthi S, Sumitha R, Varghese AM, Ashok S, Chandrasekhar Sagar BK, Sathyaprabha TN, et al. Brain-derived neurotrophic factor facilitates functional recovery from ALS-cerebral spinal fluid-induced neurodegenerative changes in the NSC-34 motor neuron cell line. Neurodegener Dis. 2017:17:44-58

15. Gungen GO, Ardic F, Findikoglu G, Rota S. Effect of mud compress therapy on cartilage destruction detected by CTX-II in patients with knee osteoarthritis. J Back Musculoskelet Rehabil. 2016;29:429-38.

16. Zayed M, Newby S, Misk N, Donnell R, Dhar M. Xenogenic implantation of equine synovial fluid-derived mesenchymal stem cells leads to articular cartilage regeneration. Stem Cells Int. 2018;2018:1073705.

17. Schuermann Y, Siddappa D, Pansera M, Duggavathi R. Activated receptor tyrosine kinases in granulosa cells of ovulating follicles in mice. Mol Reprod Dev. 2018;85:316-24

18. Mullen LM, Best SM, Ghose S, Wardale J, Rushton N, Cameron RE. Bioactive IGF-1 release from collagen-GAG scaffold to enhance cartilage repair in vitro. J Mater Sci Mater Med. 2015;26(1):5325.
19. Correa D, Somoza RA, Lin P, Greenberg S, Rom E, Duesler L, et al. Sequential exposure to fibroblast growth factors (FGF) 2, 9 and 18 enhances hMSC chondrogenic differentiation. Osteoarthritis Cartilage. 2015;23:443-53.

20. Rangbulla V, Nirola A, Gupta M, et al. Salivary IgA, Interleukin-1 $\beta$ and MMP-8 as salivary biomarkers in chronic periodontitis patients. Chin J Dent Res. 2017;20:43-51.

21. Naik SP, Mahesh PA, Jayaraj BS, Madhunapantula SV, Jahromi SR, Yadav MK. Evaluation of inflammatory markers interleukin-6 (IL-6) and matrix metalloproteinase-9 (MMP-9) in asthma. J Asthma. 2017;54:584-93.

22. Wang $X$, Hunter D, Xu J, Ding C. Metabolic triggered inflammation in osteoarthritis. Osteoarthritis Cartilage. 2015;23:22-30.

23. Kohno K, Itoh S, Hanai A, Takii T, Fujiwara T, Onozaki K, et al. dentification of matrix metalloproteinase 9-interacting sequences in staphylococcal superantigen-like protein 5. Biochem Biophys Res Commun. 2018:497:713-8.

24. Saravani S, Yari D, Saravani R, Azadi AC. Association of COL4A3 (rs55703767), MMP-9 (rs17576) and TIMP-1 (rs6609533) gene polymorphisms with susceptibility to type 2 diabetes. Biomed Rep. 2017;6:329-34

25. Martineau X, Abed É, Martel-Pelletier J, Pelletier JP, Lajeunesse D. Alteration of Wnt5a expression and of the non-canonical Wnt/PCP and Wnt/PKC-Ca2+ pathways in human osteoarthritis osteoblasts. PloS One. 2017:12:e0180711

26. Lv H, Chen W, Yuwen P, Yang N, Yan X, Zhang Y. Multivariate analysis of factors related to radiographic knee osteoarthritis based on the comparison between football players and matched nonsportsmen. Int Orthop. 2018;42: 519-27.

27. Petersen KK, Arendt-Nielsen L, Finocchietti S, Hirata RP, Simonsen O, Laursen $M B$, et al. Age interactions on pain sensitization in patients with severe knee osteoarthritis and controls. Clin J Pain. 2017:33:1081-7.

\section{Publisher's Note}

Springer Nature remains neutral with regard to jurisdictional claims in published maps and institutional affiliations.
Ready to submit your research? Choose BMC and benefit from:

- fast, convenient online submission

- thorough peer review by experienced researchers in your field

- rapid publication on acceptance

- support for research data, including large and complex data types

- gold Open Access which fosters wider collaboration and increased citations

- maximum visibility for your research: over $100 \mathrm{M}$ website views per year

At $\mathrm{BMC}$, research is always in progress.

Learn more biomedcentral.com/submissions 